\title{
Two-stage product design selection by using PROMETHEE and Taguchi method: A case study
}

\author{
Crnjac, M. ${ }^{a}{ }^{\mathrm{a}}$, Aljinovic, A. ${ }^{a}$, Gjeldum, N. ${ }^{a}$, Mladineo, M. $^{\mathrm{a}}$ \\ ${ }^{a}$ University of Split, Faculty of Electrical Engineering, Mechanical Engineering and Naval Architecture, Split, Croatia
}

\begin{abstract}
A B S T R A C T
The main goal of this paper was to introduce the methodology for product design selection. The proposed methodology combines two classical methods to find the most appropriate design for the new product, through a reduced number of alternatives (product variants) and experiments for the selection process. In the first stage, the multi-criteria decision-making method, PROMETHEE was used for selecting the most suitable design, according to the chosen preferences and criteria. In the second stage, the Taguchi method was used in order to define the most appropriate parameters for selected suitable design. The fundamental scientific contribution of this paper refers to a benefit introduced by combining these methods. This benefit is related to the reduction of product development time which has a significant effect on manufacturing process time due to the high market pressure. The proposed methodology was applied to find the appropriate table design for CNC milling machine located in the Lean Learning factory. However, this is just one case study to present the proposed methodology which can be applied for other optimization of other product designs. Before applying the proposed methodology for this case study, the methodology is validated on a simple example.
\end{abstract}

\section{ARTICLE INFO}

Keywords:

Learning factory;

Lean manufacturing;

Design optimization;

PROMETHEE method;

Taguchi method;

*Corresponding author:

mcrnjac@fesb.hr

(Crnjac, M.)

Article history:

Received 9 October 2018

Revised 25 February 2019

Accepted 27 February 2019

\section{References}

[1] Ishizaka, A., Nemery, P. (2013). Multi-criteria decision analysis: Methods and software, John Wiley \& Sons, New Delhi, India, doi: 10.1002/9781118644898.

[2] Govindan, K., Rajendran, S., Sarkis, J., Murugesan, P. (2015). Multi criteria decision making approaches for green supplier evaluation and selection: A literature review, Journal of Cleaner Production, Vol. 98, 66-83, doi: 10.1016 Li.jclepro.2013.06.046.

[3] Mladineo, M., Veza, I., Gjeldum, N. (2017). Solving partner selection problem in cyber-physical production networks using the HUMANT algorithm, International Journal of Production Research, Vol. 55, No. 9, 2506-2521, doi: 10.1080/00207543.2016.1234084.

[4] Mardani, A., Jusoh, A., Zavadskas, E.K. (2015). Fuzzy multiple criteria decision-making techniques and applications - Two decades review from 1994 to 2014, Expert Systems with Applications, Vol. 42, No. 8, 4126-4148, doi: 10.1016/j.eswa.2015.01.003.

[5] Avikal, S., Mishra, P.K., Jain, R. (2014). A Fuzzy AHP and PROMETHEE method-based heuristic for disassembly line balancing problems, International Journal of Production Research, Vol. 52, No. 5, 1306-1317, doi: 10.1080 /00207543.2013.831999.

[6] Peko, I., Gjeldum, N., Bilić, B. (2018). Application of AHP, fuzzy AHP and PROMETHEE method in solving additive manufacturing process selection problem, Tehnički Vjesnik - Technical Gazette, Vol. 25, No. 2, 453-461, doi: $10.17559 /$ TV-20170124092906.

[7] Vinodh, S., Girubha, R.J. (2012). PROMETHEE based sustainable concept selection, Applied Mathematical Modelling, Vol. 36, No. 11, 5301-5308, doi: 10.1016/i.apm.2011.12.030.

[8] Can, A., Ünüvar, A. (2017). Optimization of process parameters in drilling of SMC composites using Taguchi method, Tehnički vjesnik - Technical Gazette, Vol. 24, No. 2, 435-442, doi: 10.17559/TV-20160103215256. 
[9] Chang, H.-C., Chen, H.-Y. (2014). Optimizing product form attractiveness using Taguchi method and TOPSIS algorithm: A case study involving a passenger car, Concurrent Engineering, Vol. 22, No. 2, 135-147, doi: 10.1177/ $1063293 X 13520317$.

[10] Oztekin, A., Iseri, A., Zaim, S., Nikov, A. (2013). A Taguchi-based Kansei engineering study of mobile phones at product design stage, Production Planning \& Control, Vol. 24, No. 6, 465-474, doi: 10.1080/09537287.2011. $\underline{633575}$.

[11] Mladineo, M., Celar, S., Celent, L., Crnjac, M. (2018). Selecting manufacturing partners in push and pull-type smart collaborative networks, Advanced Engineering Informatics, Vol. 38, 291-305, doi: 10.1016/j.aei.2018.08.001.

[12] Gjeldum, N., Mladineo, M., Veza, I. (2016). Transfer of model of innovative smart factory to Croatian economy using lean learning factory, Procedia CIRP, Vol. 54, 158-163, doi: 10.1016/i.procir.2016.06.096.

[13] Abele, E., Metternich, J., Tisch, M., Chryssolouris, G., Sihn, W., ElMaraghy, H., Hummel, V., Ranz, F. (2015). Learning factories for research, education, and training, Procedia CIRP, Vol. 32, 1-6, doi: 10.1016/i.procir.2015.02.187.

[14] Prinz, C., Morlock, F., Freith, S., Kreggenfeld, N., Kreimeier, D., Kuhlenkötter, B. (2016). Learning factory modules for smart factories in Industrie 4.0, Procedia CIRP, Vol. 54, 113-118, doi: 10.1016/j.procir.2016.05.105.

[15] Herrmann, C., Schmidt, C., Kurle, D., Blume, S., Thiede, S. (2014). Sustainability in manufacturing and factories of the future, International Journal of Precision Engineering and Manufacturing-Green Technology, Vol. 1, No. 4, 283292, doi: $10.1007 / \mathrm{s} 40684-014-0034-\mathrm{z}$.

[16] Rao, S.S. (2017). The finite element method in engineering, (6th edition), Butterworth-Heinemann, Oxford, UK, doi: 10.1016/C2016-0-01493-6.

[17] Özkal, F.M., Cakir, F., Arkun, A.K. (2016). Finite element method for optimum design selection of carport structures under multiple load cases, Advances in Production Engineering \& Management, Vol. 11, No. 4, 287-298, doi: 10.14743/apem2016.4.227.

[18] Wang, Y., Lu, Y.J., Si, C.D., Sun, T.C. (2017). Finite element analysis for rutting prediction of asphalt concrete pavement under moving wheel load, International Journal of Simulation Modelling, Vol. 16, No. 2, 229-240, doi: 10.2507/IJSIMM16(2)4.374.

[19] Sari, T., Timor, M. (2016). Integrated supplier selection model using ANP, Taguchi loss function and PROMETHEE methods, Journal of Applied Quantitative Methods, Vol. 11, No. 1, 19-34.

[20] Brans, J.-P., De Smet, Y. (2016). PROMETHEE methods, In: Greco, S., Ehrgott, M., Rui Figueira, J. (eds.), Multiple Criteria Decision Analysis, Springer, New York, USA, 187-219, doi: 10.1007/978-1-4939-3094-4.

[21] Behzadian, M., Kazemzadeh, R.B., Albadvi, A., Aghdasi, M. (2010). PROMETHEE: A comprehensive literature review on methodologies and applications, European Journal of Operational Research, Vol. 200, No. 1, 198-215, doi: 10.1016/j.ejor.2009.01.021.

[22] Brans, J.-P., Mareschal, B. (2005). PROMETHEE methods, In: Figueira, J., Greco, S., Ehrgott, M. (eds.), Multiple Criteria Decision Analysis: State of the Art Surveys, Vol. 78, Springer, New York, USA, 163-186, doi: 10.1007/0387-23081-5_5.

[23] Brans, J.P., Vincke, Ph. (1985). Note - A preference ranking organization method (The PROMETHEE method for Multiple Criteria Decision-Making), Management Science, Vol. 31, No. 6, 647-784, doi: 10.1287/mnsc.31.6.647.

[24] Phadke, M.S. (1995). Quality engineering using robust design, Prentice Hall PTR, New Jersey, USA.

[25] Li, Y., Zhu, L. (2017). Optimisation of product form design using fuzzy integral-based Taguchi method, Journal of Engineering Design, Vol. 28, No. 7-9, 480-504, doi: 10.1080/09544828.2017.1346239.

[26] Genç, T., Dinçer, S.E. (2013). Visual analysis for multi criteria decision problems by PROMETHEE method and gaia plane: An application, determine the level of regional socio-economic development in Turkey, Trakya University Journal of Social Science, Vol. 15, No. 2, 111-130.

[27] Mareschal, B. The PROMETHEE - GAIA FAQ, from http://www.promethee-gaia.net/, accessed July 11, 2018.

[28] Cardinal, R.N., Aitken, M.R.F. (2013). ANOVA for the behavioral sciences researcher, Psychology Press, New York, USA, doi: $10.4324 / 9780203763933$. 


\section{APEM}

\title{
Dvostopenjska izbira oblikovanja izdelkov z uporabo metod PROMETHEE in Taguchi: Študija primera
}

\author{
Crnjac, M. ${ }^{a}{ }^{a}{ }^{*}$, Aljinovic, A. ${ }^{a}$, Gjeldum, N. ${ }^{a}$, Mladineo, M. ${ }^{a}$ \\ ${ }^{a}$ University of Split, Faculty of Electrical Engineering, Mechanical Engineering and Naval Architecture, Split, Croatia
}

\section{POVZETEK}

Glavni cilj tega prispevka je predstaviti metodologijo izbire oblikovanja izdelkov. Predlagana metodologija združuje dve klasični metodi, ki omogočata poiskati najustreznejšo zasnovo novega izdelka, z zmanjšanim številom alternativ (različic izdelka) in eksperimentov za izbirni postopek. V prvi fazi je bila za izbiro najprimernejše zasnove $\mathrm{v}$ skladu z izbranimi željami in merili uporabljena večkriterijska metoda odločanja, PROMETHEE. V drugi fazi je bila za določitev najprimernejših parametrov za izbrano zasnovo uporabljena metoda Taguchi. Temeljni znanstveni prispevek tega prispevka se nanaša na korist, ki jo uvaja kombinacija obeh metod. Ta korist je povezana s skrajšanjem časa razvoja izdelka, ki zaradi velikega tržnega pritiska pomembno vpliva na čas izdelave. Predlagana metodologija je bila uporabljena za iskanje ustrezne zasnove mize za CNC rezkalne stroje, ki se nahajajo v vitki didaktični tovarni. Vendar je to le ena študija primera, ki predstavlja predlagano metodologijo, ki jo je mogoče uporabiti tudi za optimizacije drugih modelov izdelkov. Pred uporabo predlagane metodologije za to študijo primera je metodologija potrjena na preprostem primeru.

\section{PODATKI O ČLANKU}

Ključne besede:

Didaktična tovarna;

Vitka proizvodnja; Optimizacija oblikovanja; PROMETHEE metoda; Taguchi metoda

*Kontaktna oseba: mcrnjac@fesb.hr (Crnjac, M.)

Zgodovina članka:

Prejet 9. oktobra 2018

Popravljen 25. februarja 2019

Sprejet 27. februarja 2019 